\title{
Can I intrude?
}

\section{What is the magnitude of true incisor intrusion that can be obtained attained during orthodontic treatment?}

\author{
Ng J, Major PW, Heo G, Flores-Mir C. \\ True incisor intrusion attained during orthodontic treatment: a \\ systematic review and meta-analysis. Am J Orthod Dentofacial \\ Orthop 2005; 128:212-219
}

Data sources Studies were identified using Medline, PubMed, Medline In-Process and Other Non-Indexed Citations, all evidence-based medicine reviews (Cochrane Database of Systematic Reviews, ACP (American College of Physicians) Journal Club, Database of Reviews of Effectiveness (DARE) and CCTR (Cochrane Controlled Trials Register), Embase, Web of Science and LILACS(Latin American and Caribbean Health Sciences).

Study selection Studies included were clinical trials in humans where the intrusion of permanent incisors was determined using cephalometric radiograph superimpositions.

Data extraction and synthesis A qualitative synthesis of all included studies was conducted, along with a fixed-effects meta-analysis of two studies that employed the same treatment method.

Results Of 28 articles meeting the initial selection criteria, only four were included because of methodological issues with the others. Two studies that used the segmented arch technique were included in a fixed-effects meta-analysis to evaluate the amount of incisor intrusion. The combined mean estimates were $1.46 \mathrm{~mm}$ (95\% confidence interval, 1.05-1.86) for the maxillary incisor and $1.90 \mathrm{~mm}$ (95\% confidence interval, 1.22-2.57) of intrusion for the mandibular incisor.

Conclusions True incisor intrusion is achievable in both arches, but the clinical significance of the magnitude of true intrusion as the sole treatment option is questionable for patients with severe deep bite. In non-growing patients, the segmented arch technique can produce $1.5 \mathrm{~mm}$ of incisor intrusion in the maxillary arch and $1.9 \mathrm{~mm}$ in the mandibular arch.

\section{Commentary}

There is a paradox in clinical orthodontics, that the simple and most common malocclusion is the one that is most difficult to treat. Deep bite falls into this category and, despite rapid clinical and technological advances, it still constitutes an enigma for which clear answers are lacking. This systematic review and meta-analysis is a bold attempt to resolve that tangled issue and present a clear evidence-based perspective. The only problem is that there is a paucity of evidence and, after the initial inclusion criteria and a further refined search, four studies met the criteria of the review and only two of those could contribute to a meta-analysis - a challenging task at best for the reviewers.
The problem with quantifying true incisor intrusion lies in the specifics of isolating the effects of treatment from any growth- or age-related remodelling changes and the points of reference. Incisal edges and root apices could well be misleading because inclination changes could give a false-positive result. The ability to measure the absolute intrusion would need a centroid point of the incisors and a reference plane able to take into account the actual change in position of the incisors.

The aims of this review are specific in terms of quantifying the amount of true incisor intrusion and evaluating its clinical significance. The search strategy is thorough and in view of the above factors the reviewers have done well to specify stringent inclusion criteria for study inclusion.

The study by Hans et al. poses a problem for deriving conclusions because it is the only one in the review dealing with a group of patients in prepubertal growth. The use of orthopaedic/ functional appliances would probably have less of a dental effect than a growth modulation or modification effect. Filtering out the growth- and agerelated changes would be difficult.

The other issue that arises in the review is that selected studies use different appliances for intrusions so data synthesis is therefore problematic. The fact that the segmented arch technique is more successful in achieving true incisor intrusion is not surprising since the force levels and the direction of force must be precisely forecast and monitored to achieve intrusion. The meta-analysis of incisor intrusion with the segmented arch technique, however, yields a positive clinical bottom line.

The fact that about $1.5 \mathrm{~mm}$ of maxillary incisor intrusion and $1.9 \mathrm{~mm}$ of mandibular intrusion is routinely possible would satisfy the clinician. The meta-analysis is based only on two studies and therefore caution should be exercised in interpretation of its results because of publication bias, methodological heterogeneity and differences in quality between the studies. Nevertheless, the outcome is very clear - the segmented arch technique is effective in achieving true intrusion, so relevant in adult orthodontic treatment.

The reviewers have addressed an issue that is pertinent and relevant in day-to-day clinical practice with a mature, systematic and sound evidence-based approach. The art within the science of clinical orthodontics stands reaffirmed. In a world where the prevalent direction is towards preadjustment and preformed materials, the need seen here for critical orthodontic reasoning and skills when managing the segmented arch endorses how, ultimately, it is the orthodontist and not the manufacturer who determines the success of treatment.

\section{Anmol Kalha \\ Department of Orthodontics, Centre for Evidence Based Dentistry, College of Dental Sciences, Davangere, Karnataka, India}

Evidence-Based Dentistry (2007) 8, 17. doi:10.1038/sj.ebd.6400469 